\title{
A simple clinical score to identify likely hepatitis B vaccination non-responders - data from a retrospective single center study
}

\author{
Marc A. Meier ${ }^{1}$ and Christoph T. Berger ${ }^{1,2^{*}}$ (D)
}

\begin{abstract}
Background: About 10\% of Hepatitis B vaccinated individuals mount no protective antibody levels against the hepatitis B surface antigen (HBs-Ag). Older age at primary immunization, obesity and smoking have previously been reported as risk factors associated with vaccine non-response. Here we tested whether these factors alone may allow selecting subjects that benefit from individualized immunization schedules.

Methods: Retrospective database analysis screening > 15,000 individual anti-HBs-lgG measurements. Non-responders (NR; anti-HBs-lgG < $10 \mathrm{IU} / \mathrm{L}$ ) and low-responders (LR; anti-HBs-lgG 10-100 IU/L) were identified. Vaccine type, demographics, lifestyle, and immunological factors (leucocyte subset counts) were compared between NR, LR, and responders (R).

Results: We identified $113 \mathrm{LR} / \mathrm{NR}$ and compared them to 134 vaccine responders. We confirmed higher median age at primary vaccination (24.0 (R) vs. 30.5 (NR) vs. 31 (LR) years, $p=0.001)$, higher median BMl $\left(23.2 \mathrm{~kg} / \mathrm{m}^{2}\right.$ (R) vs. $23.4 \mathrm{~kg} / \mathrm{m}^{2}$ (NR) vs. $25.1 \mathrm{~kg} / \mathrm{m}^{2}(\mathrm{LR}), \mathrm{p}=0.001$ ) and being a smoker (\% smokers: $30.8 \%$ (R) vs. $57.1 \%$ (NR) vs. $52.5 \%(\mathrm{LR}), p=0.01$ ) as factors negatively associated with anti-HBs-IgG levels. In a ROC analysis including these factors in a 6-point score, a high score predicted non-response with a specificity of $85 \%$ but at low sensitivity (47\%).

Conclusion: A simple clinical risk score based on age, obesity, and smoking identifies individuals with a high likelihood of vaccine failure. Non-responders with a low score are candidates for in-depth analyses to better understand the immunological causes of HBV vaccine non-response.
\end{abstract}

Keywords: Hepatitis B, Vaccine, Non-response, HBs, Antibodies, Smoking, Age, BMI, Risk score

\section{Background}

Chronic Hepatitis B virus (HBV) infection affects about 400 million individuals worldwide, and cumulatively accounts for about 1 million deaths annually [1]. Vaccination against HBV is safe and very effective in preventing HBV infection and its complications [2]. Since the early 1990s, high-risk

\footnotetext{
* Correspondence: christoph.berger@usb.ch

${ }^{1}$ Vaccination Clinic, Medical Outpatient Unit, University Hospital Basel, Petersgraben 4, 4031 Basel, Switzerland

${ }^{2}$ Translational Immunology, Department of Biomedicine, University of Basel, Basel, Switzerland
}

(c) The Author(s). 2020 Open Access This article is licensed under a Creative Commons Attribution 4.0 International License, which permits use, sharing, adaptation, distribution and reproduction in any medium or format, as long as you give appropriate credit to the original author(s) and the source, provide a link to the Creative Commons licence, and indicate if changes were made. The images or other third party material in this article are included in the article's Creative Commons licence, unless indicated otherwise in a credit line to the material. If material is not included in the article's Creative Commons licence and your intended use is not permitted by statutory regulation or exceeds the permitted use, you will need to obtain permission directly from the copyright holder. To view a copy of this licence, visit http://creativecommons.org/licenses/by/4.0/ The Creative Commons Public Domain Dedication waiver (http://creativecommons.org/publicdomain/zero/1.0/) applies to the data made available in this article, unless otherwise stated in a credit line to the data.

populations -especially health care workers- have been systematically vaccinated. More recently, vaccination efforts have been extended and many national vaccination programs now recommend HBV vaccination for the general population. The currently available HBV vaccine preparations use recombinant hepatitis B surface antigen ( $\mathrm{HBsAg})$ as the antigen. Vaccination induces an antibody-based protective immunity targeting the $\mathrm{HBsAg}[3,4]$.

Primary immunization protects at least two decades in the vast majority of immunocompetent individuals [5]. An anti-HBs antibody titer above $100 \mathrm{IU} / \mathrm{L}$ is considered 
protective, whereas titers below $10 \mathrm{IU} / \mathrm{L}$ confer no reliable protection from infection. Accordingly, vaccine recipients are graded into 'non-responders' $(<10 \mathrm{IU} / \mathrm{L})$, 'low-responders' (10-100 IU/L), and 'responders' (> $100 \mathrm{IU} / \mathrm{L})$, respectively [6]. Large vaccination studies suggested nonresponder rates in the range of 5 to $10 \%$ of all $\mathrm{HBV}$ vaccinated healthy individuals vaccinated using the standard vaccination regimen [2]. Several epidemiological studies have established that age at immunization, overweight, gender, smoking status, co-morbidities and immunosuppression affect the vaccine response [6-15]. In many of the subjects with these 'risk factors', protective immunity can be achieved by repeated additional doses ('booster') [3]. The reason for the failure to adequately respond to HBV vaccination in subjects without classical risk factors has been attributed to genetic factors (MHC), reduced $\mathrm{T}$ cell activation or failure of the $\mathrm{T}$ cells to recognize the $\mathrm{HBs} \mathrm{Ag}$ ('hole in the repertoire') [16]. Here, we studied a group of hepatitis B vaccine non-responders and tested, if a simple score based on age, and -the potentially modifiable life-style factorsBMI and smoking status may allow selecting subjects with a high likelihood for vaccine failure.

\section{Methods}

\section{Vaccine recipient cohorts and screening strategy}

The local Ethical committee (EKNZ-265/12) approved the study. We performed a retrospective database screening of HBs-IgG test results collected between 1995 and 2012. We limited our analysis on 'potential non-responders' from the medical outpatient clinic, the vaccination clinic and occupational health service (OHS), in order to exclude subjects with relevant comorbidities. We excluded subjects with only a single anti-HBs-IgG measurement, and we divided the remaining into 'potential non-responders' (all measurements < $100 \mathrm{IU} / \mathrm{L}$ ) vs. 'responders' (at least one measurement $>100 \mathrm{IU} / \mathrm{L}$ ). For the patient's chart review of all 'potential non-responders' we excluded subjects with (i) no chart available; (ii) incomplete primary vaccination schedules; (iii) obvious immunosuppression (i.e. chemotherapy, HIV, active cancer, immunosuppressive drugs); (iv) documented titers $>100 \mathrm{IU} / \mathrm{L}$ in the chart, (v) age $>60$ at primary immunization, or (v) natural $\mathrm{HBV}$ infection. As a control group, 134 subjects with well-documented vaccination schedules and demographic factors were selected from the responder pool. The screening and subject selection process is summarized in Figure S1.

Data collection for all studied subjects included: antiHBs-IgG levels, vaccine preparation/brand, vaccination schedule, demographic and lifestyle factors (age, gender, BMI, smoking habits). Besides, total white blood cell count (WBC), neutrophil count, and lymphocyte count were also extracted from the charts, if these were available within a time window of 6 months before and/or after vaccination.
Mean values were calculated in cases where several time points were available within the defined time window.

\section{Statistical analyses}

We compared continuous variables between two groups using an unpaired t-test. Binary data were analyzed using chi-square tests. For comparisons of more than two groups one-way ANOVA (Kruskal-Wallis) was used followed by correction for multiple comparisons (Dunn's correction). Logistic regression analysis was performed using JMP software (V11, Institute). All statistical analyses, with the exception of the logistic regression, were performed using Prism Software (V6.0b, GraphPad Software).

\section{Results}

Characterization of vaccine responders and non-responders We identified 40 'non-responders' and 73 'low responders' that we compared to 134 'responders'. AntiHBs-IgG levels in the responders were highly variable covering, almost $2.5 \mathrm{log}$ differences between the extremes (Figure S2). Non-responders received a median of six HBV vaccine doses (range 3-12) compared to four (range $3-7)$ in the responders $(p<0.001)$. Notably, 23.7\% of the non-responders and $23.3 \%$ of the low-responders received seven or more vaccinations (Figure S2).

Gender distribution was equal between the three groups (Fig. 1a and Table S1). At the time of the basic immunization, non- and low-responders were significantly older than the responders (Fig. 1b). BMI was significantly higher in low-responders compared to responders (median $25.1 \mathrm{~kg} / \mathrm{m}^{2}$ vs. $23.2 \mathrm{~kg} / \mathrm{m}^{2}, p<0.001$ ), but not between the responder and the non-responder group (Fig. 1c). About 1 out of 3 non-responders (29.6\%) and 1 out of 4 low-responders (24.5\%) had a BMI above $28 \mathrm{~kg} / \mathrm{m}^{2}$, while this was only the case in $4.7 \%$ of the responders. Low- and non-responders were comparably enriched for smokers, but smokers in the non-responder group were almost exclusively "heavy smokers', defined as $\geq 10$ cigarettes per day (Fig. 1d).

Various vaccine types were available during the study period (Table S1). Data on the vaccine used for the baseline immunization was available for all non-responders $(n=113)$ and $59 \%$ of the responders $(n=73)$. We found that the vaccines used in the groups were very comparable, with Engerix being the most frequently applied in both groups (Fig. 1e). The only vaccine that was used more frequently in the non-responder group ( $8 \%$ vs. $2.7 \%)$ was Heprecomb ${ }^{\circ}$. Notably, Heprecomb $b^{\circ}$ contains a low HBs antigen dose and is the preparation with the lowest adjuvant content (aluminum hydroxide) (Table S2).

Leucocyte or lymphocyte counts were only available for a small subset of the cohort ( $n=65$ for the leucocyte counts; $n=38$ for the lymphocyte counts), preventing meaningful analyses. We found, however, that neither relative leucopenia, neutropenia, or lymphopenia were associated with 

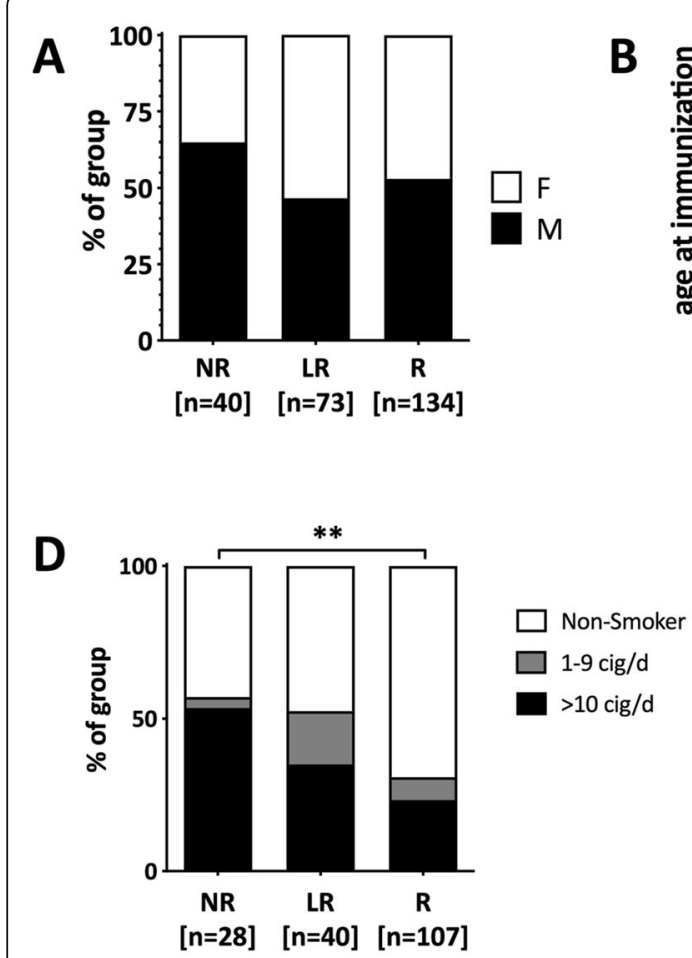
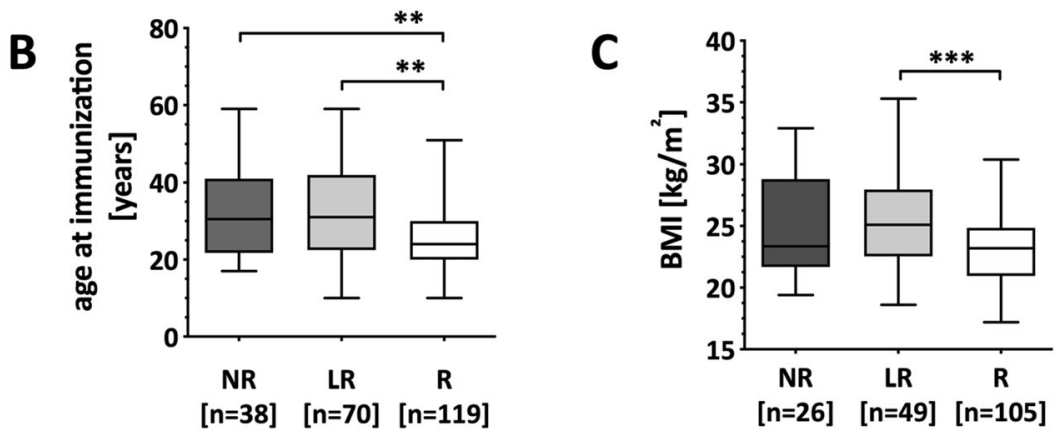

Fig. 1 Association of demographic and lifestyle factors with vaccine response status. Distribution of sex in \% within each group (a), age at the first HBV vaccination (b), Body Mass Index (BMI) (c), and \% smokers per group (d) is shown. Kruskal-Wallis test was used to compare the three groups. Smokers were subdivided into light (0-9 cigarettes/day; grey) or heavy ( $\geq 10$ cigarettes/day; black) smokers. (e) Distribution of vaccine preparation/type in responders (white bars) and low-/non-responders (black bars). ${ }^{* *} p<0.01,{ }^{* *} p<0.001$

vaccine non-response. Contrarily, the low-/non-responders had the highest leucocyte, neutrophil, and lymphocyte counts (Fig. S3).

In summary, the analysis of the demographic and lifestyle factors on the vaccine response confirmed a negative impact of age at immunization, overweight, and smoking.

\section{High specificity, but low sensitivity of a risk score to predict non-response}

We next tested if a risk score would identify subjects with a high risk of $\mathrm{HBV}$ vaccine non-response. Logistic regression analysis confirmed the negative impact of the three risk factors: age, smoking and high BMI. BMI showed the strongest association $(p=0.0005)$, followed by smoking status $(p=0.011)$, and age at vaccination $(p=0.022)$. We calculated a score using a study sample subset for which data on all of these three parameters were available (i.e. $R=94$, $\mathrm{NR}=24, \mathrm{LR}=36$ ). Each variable was categorized in three clinically relevant groups: i) normal BMI (0 points), BMI > $25-30 \mathrm{~kg} / \mathrm{m}^{2}$ (1 point), and BMI $>30 \mathrm{~kg} / \mathrm{m}^{2}$ (2 points); ii) Age at primary hepatitis B immunization $<30$ (0 points), 30-40 (1point), and > 40 ( 2 points); and iii) non-smoker $(0$ points), $1-9$ cigarettes a day ( 1 point), and $\geq 10$ cigarettes a day ( 2 points), resulting in a potential score between 0 and
6. Vaccine responders had a median score of 1 (IQR 0-2), low-responders a median score of 2 (IQR 1-3), and nonresponders a median score of 2.5 (IQR 1-4) (Fig. 2a). Only about $5 \%$ of the responders, but $35 \%$ of the low-/non-responders had a score of 4 or higher. In contrast, about $85 \%$ of the responders had a score of 2 or less, while this was the case in less than half of the low-/non-responders combined. In a ROC curve, a cut-off of $\geq 2$ score points yielded a sensitivity of $71.7 \%$ and specificity of $64.9 \%$. Specificity was high $(86 \%)$ at a higher cut-off of $\geq 4$ score points at the cost of a low sensitivity (47\%) (Fig. 2b).

When we performed the same analysis separately for subjects that received $\leq 4$ vs. those who received $\geq 5$ vaccinations, we found that the AUC was substantially lower in those who received $\geq 5$ vaccinations ( 0.79 vs. 0.66) (data not shown). This suggests that in subjects that mount no protective titers following repeated boostering (i.e. $\geq 5$ vaccinations) the risk factors age, BMI, and smoking status were less relevant.

\section{Discussion}

HBV vaccination results in protective antibody titers in about $90 \%$ of the vaccinated subjects. Anti-HBs antibody titer measurements are clinically available, but due to 


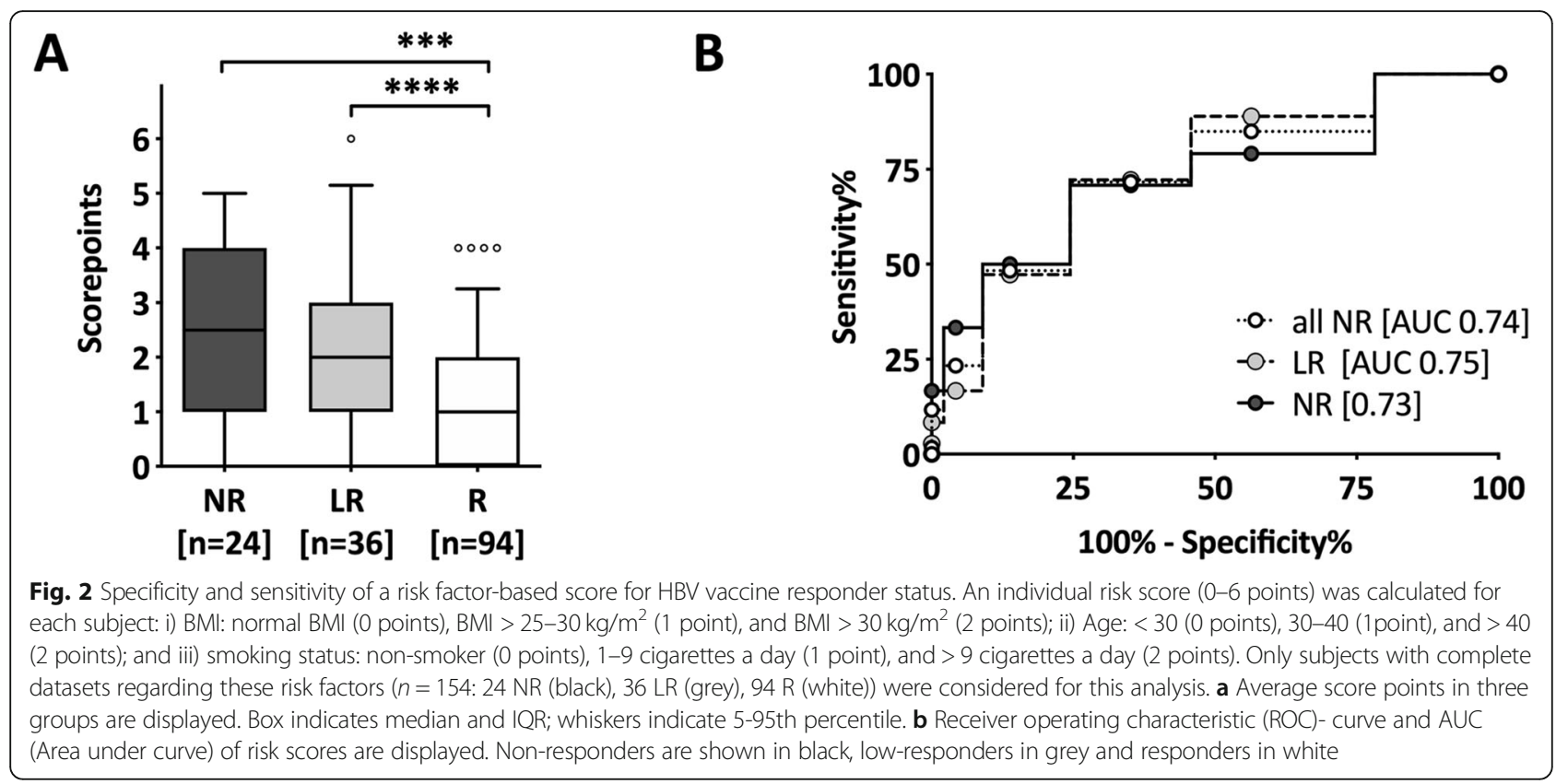

low cost-effectiveness only recommended for high-risk populations, specifically health care workers. However, on an individual basis, every vaccinated subject would want to know whether they are fully protected. Recent epidemiological data indicate that currently, other populations than health care workers are at the highest risk to contract HBV [17]. Hence, there is a need to identify non-responders that could be specifically tested and vaccinated using individualized vaccination protocols [7]. Overall, this would allow increasing the population coverage of immunity and thereby reducing the disease prevalence in a population.

Here we performed a retrospective, single-center study to test whether age, BMI and smoker status could be used -alone or in combination- to predict HBV vaccination non-response. We confirmed that higher age at vaccination, smoking, and a high BMI associated with a higher risk for non-response [10-14]. About 1 out of 3 nonresponders (or 29.6\%) and 1 out of 4 low-responders $(24.5 \%)$ had a BMI above $28 \mathrm{~kg} / \mathrm{m}^{2}$, while this was only the case in $4.7 \%$ of the responders. There are several potential explanations for this finding: i) the amount of $\mathrm{HBs} \mathrm{Ag}$ is too low in relation to the body mass, ii) there is a substantial immunological dysfunction in the obese [18], or iii) the needles are too short to reach the muscle, thus resulting in subcutaneous vaccination associated with blunted responses [7]. The negative effect of older age, may be explained with the progressive immune dysfunction occurring in an aging organism. Interestingly, this immune dysfunction seems to be evolving already in middleaged subjects, as we excluded all vaccinees older than 60 years. Finally, we confirmed that smoking seems to have a strong and dose-dependent impact on HBV vaccine response in a larger series of non-/low-responders [11, 14]. In contrast to others, we observed an equal gender distribution in responders and non-responders. Concomitant disease has been suggested as another risk factor for lower seroconversion to the HBV vaccine. In our study, we focused on subjects $<60$ years old without obvious immunosuppression. Since co-morbidities were not prospectively assessed, we cannot exclude they may have contributed to vaccine failure in some instances.

Using quantification of leucocytes and leucocyte subsets, we could exclude that absolute (immune cell deficiency) or relative immunocytopenia (low numbers within the normal range for any of the immune cell subsets) are frequent causes of poor vaccine response status. It is, however, of little surprise that such crude measurements fail to reflect non-response. Thus, more in-depth immunological analyses would be necessary that should preferentially be performed on non-responders lacking any of the other risk factors for non-response.

We calculated a very simple risk score integrating the three identified risk factors: age, BMI and smoker status. A high score ( $\geq 4$ points) allowed the identification of nonresponders with high specificity (86.2\%), but at the cost of low sensitivity (46.7\%). In other words, if the score would be calculated at the time of vaccination and HBs-IgG would be determined after primary immunization, we would expect that 17 out of 20 tested subjects with a score $\geq 4$ would be LR or NR. Thus, our risk score could be used to detect at least a subgroup of non-responders. This finding may justify targeted anti-HBs-IgG measurements and individualized vaccination strategies in these selected subjects with a high score. That the risk score 
imperfectly predicts non-responders is not surprising, as additional risk factors such as HLA type or hyporeactive $\mathrm{T}$ cells have been described as risk factors for HBV vaccination non-response, but were not addressed in our study.

Different strategies have been suggested to enhance the immune response to hepatitis $B$ vaccination, including additional booster doses, an increased HBs antigen dose, intradermal vaccinations or the use of adjuvanted vaccines [7, 19-21]. Three doses of the 3'-deacylated monophosphoryl lipid A (3D-MPL) and alum containing AS04-adjuvanted HBV vaccine resulted in seroprotection in $98 \%$ of previous non-responders (HBs-IgG $<10$ $\mathrm{U} / \mathrm{L}$ ) compared to only $68 \%$ in subjects that received three additional standard vaccine doses [21]. Very recently, a Dutch multicenter study compared the efficacy of additional three doses $(0,1,2$ months) of either the standard $\mathrm{HBV}$ vaccine, an HBV/HAV combination vaccine, a double dose vaccine ( $40 \mu \mathrm{g} \mathrm{HBsAg})$ or the AS04adjuvanted vaccine $(20 \mu \mathrm{g} \mathrm{HBsAg})$ in previous nonresponders. Percentage of responders was $67 \%$ in the standard vaccine group compared to $80 \%$ in the $\mathrm{HBV} /$ HAV vaccine group, $83 \%$ double dose group and $87 \%$ in the AS04-adjuvanted group [22]. Whether subjects at risk for non-response identified by our score should be immunized using one of these alternative strategies remains to be tested prospectively.

The main limitations of the study include the retrospective design resulting in incomplete data collection, heterogenous time points for the anti-HBs-IgG measurements following vaccinations, and the rather small, not perfectly matched control population. A validation of the score in a larger cohort or a prospective study is warranted, since we only applied it to the training cohort. Moreover, the study population that included subjects seen at a University Hospital as well as health care workers may not be representative of other populations.

A major strength of the study is that, by using a retrospective study design, we were able to identify a substantial number $(n=113)$ of HBV vaccine non-/low-responders screening more than $15^{\prime} 000 \mathrm{HBV}$ titer measurements. In most previous studies on HBV non-response focus was on antibodies after primary vaccination. In our cohort nonresponders received in average six vaccinations, but some up to 14 . Hence, this population represents true nonresponders and may potentially be enriched for subjects with immunological or genetic factors (rather than BMI, age and smoking) driving non-response.

\section{Conclusions}

Taken together, our data provide a rational to test postvaccination anti-HBs-IgG in older, obese smokers and efforts should be put into increasing response rates in these subjects $[7,23]$. At the same time, the low sensitivity of a high cut-off (i.e. $\geq 4$ ) strongly points at other risk factors for non-response that are likely of immunological nature. Future immunological studies on HBV vaccine non-response should focus on young, non-obese nonsmokers since most likely immunological factors are the cause for the failure to respond despite repeated vaccinations in this population.

\section{Supplementary Information}

The online version contains supplementary material available at https://doi. org/10.1186/s12879-020-05634-y.

Additional file 1: Table S1. Demographics and anti-HBs levels. Table

S2. Characteristics of the vaccine types used in the study population.

Figure S1. Algorithm for the identification of HBV vaccine non-

responders. Figure S2. Vaccine specific antibody titer distribution and number of vaccinations received per group. Figure S3. Reduced vaccine responses were not linked to low immune cell counts in vaccine recipients.

\section{Abbreviations}

HBV: Hepatitis B Virus; HBs Ag: Hepatitis B surface antigen; NR: (Hepatitis B vaccine) non-responders; LR: (Hepatitis $B$ vaccine) low-responders;

R: (Hepatitis B vaccine) responders; BMI: Body Mass Index; WBC: White Blood Count; HLA: Human Leucocyte Antigen; MHC: Major Histocompatibility

Complex; ANOVA: Analysis of variance; ROC curve: Receiver operating characteristic curve; AUC: Area under the curve; HAV: Hepatitis A Virus

\section{Acknowledgements}

We thank the co-workers from the vaccination clinic and the occupational health service for helping with collecting the patient charts. We thank the statisticians from the clinical trial unit at the University Hospital for their support.

\section{Authors' contributions}

MAM collected the data. MAM and CTB designed the study, analyzed the data and wrote the manuscript. Both authors have read and approved the final version of the manuscript.

Funding

The study was done without any specific funding for this project.

Availability of data and materials

All data will be made available by the corresponding author upon request.

Ethics approval and consent to participate

The Ethics committee of North-Western and Central Switzerland approved the study (Study ID\# EKNZ-265/12).

Consent for publication

Not applicable.

Competing interests

The authors have no competing interests.

Received: 1 September 2020 Accepted: 19 November 2020

Published online: 25 November 2020

References

1. Dienstag JL. Hepatitis B virus infection. N Engl J Med. 2008;359(14):1486-500.

2. Poland GA, Jacobson RM. Clinical practice: prevention of hepatitis $B$ with the hepatitis B vaccine. N Engl J Med. 2004;351(27):2832-8.

3. Kao JH, Chen DS. Global control of hepatitis B virus infection. Lancet Infect Dis. 2002;2(7):395-403.

4. Van Damme P, Van Herck K. A review of the long-term protection after hepatitis a and B vaccination. Travel Med Infect Dis. 2007;5(2):79-84.

5. Poorolajal J, Mahmoodi M, Majdzadeh R, Nasseri-Moghaddam S, Haghdoost A, Fotouhi A. Long-term protection provided by hepatitis B vaccine and need for booster dose: a meta-analysis. Vaccine. 2010;28(3):623-31. 
6. Poland GA. Hepatitis B immunization in health care workers. Dealing with vaccine nonresponse. Am J Prev Med. 1998;15(1):73-7.

7. Launay O, van der Vliet D, Rosenberg AR, Michel ML, Piroth L, Rey D. Colin de Verdiere N, Slama L, Martin K, Lortholary O et al: safety and immunogenicity of 4 intramuscular double doses and 4 intradermal low doses vs standard hepatitis B vaccine regimen in adults with HIV-1: a randomized controlled trial. JAMA. 2011;305(14):1432-40.

8. Tedaldi EM, Baker RK, Moorman AC, Wood KC, Fuhrer J, McCabe RE, Holmberg SD. Hepatitis a and B vaccination practices for ambulatory patients infected with HIV. Clin Infect Dis. 2004;38(10):1478-84.

9. Senden TF. Response to intradermal hepatitis B vaccination: differences between males and females? Vaccine. 1990;8(6):612-3.

10. Fisman DN, Agrawal D, Leder K. The effect of age on immunologic response to recombinant hepatitis B vaccine: a meta-analysis. Clin Infect Dis. 2002;35(11):1368-75.

11. Shaw FE Jr, Guess HA, Roets JM, Mohr FE, Coleman PJ, Mandel EJ, Roehm RR Jr, Talley WS, Hadler SC. Effect of anatomic injection site, age and smoking on the immune response to hepatitis B vaccination. Vaccine. 1989;7(5):425-30.

12. Van Loveren $H$, Van Amsterdam JG, Vandebriel RJ, Kimman TG, Rumke HC, Steerenberg PS, Vos JG. Vaccine-induced antibody responses as parameters of the influence of endogenous and environmental factors. Environ Health Perspect. 2001;109(8):757-64.

13. Corrao G, Calleri M, Zotti M, Barral C, Russo R, Garella D, Moiraghi Ruggenini A. Immune response to anti-HBV vaccination: study of conditioning factors. Eur J Epidemiol. 1988;4(4):492-6.

14. Winter AP, Follett EA, McIntyre J, Stewart J, Symington IS. Influence of smoking on immunological responses to hepatitis B vaccine. Vaccine. 1994;12(9):771-2.

15. Yang S, Tian G, Cui Y, Ding C, Deng M, Yu C, Xu K, Ren J, Yao J, Li Y, et al. Factors influencing immunologic response to hepatitis $B$ vaccine in adults. Sci Rep. 2016;6:27251.

16. Roukens AH, Visser $L G$. Hepatitis $B$ vaccination strategy in vaccine low and non-responders: a matter of quantity of quality? Hum Vaccin. 2011;7(6):654-7.

17. CDC. Hepatitis B - Epidemiology and Prevention of Vaccine-Preventable Diseases. In: The Pink Book: Course Textbook. Volume 12th Edition edn; 2012.

18. Sheridan PA, Paich HA, Handy J, Karlsson EA, Hudgens MG, Sammon AB, Holland $L A$, Weir S, Noah TL, Beck MA. Obesity is associated with impaired immune response to influenza vaccination in humans. Int J Obes. 2012:36(8):1072-7.

19. Walayat S, Ahmed Z, Martin D, Puli S, Cashman M, Dhillon S. Recent advances in vaccination of non-responders to standard dose hepatitis $B$ virus vaccine. World J Hepatol. 2015;7(24):2503-9.

20. Fabrizi F, Dixit V, Messa P, Martin P. Hepatitis B virus vaccine in chronic kidney disease: improved immunogenicity by adjuvants? A meta-analysis of randomized trials. Vaccine. 2012;30(13):2295-300.

21. Jacques $P$, Moens $G$, Desombere I, Dewijngaert J, Leroux-Roels $G$, Wettendorff $M$, Thoelen $S$. The immunogenicity and reactogenicity profile of a candidate hepatitis $B$ vaccine in an adult vaccine non-responder population. Vaccine. 2002;20(31-32):3644-9.

22. Raven SFH, Hoebe C, Vossen A, Visser LG, Hautvast JLA, Roukens AHE, van Steenbergen JE. Serological response to three alternative series of hepatitis $B$ revaccination (Fendrix, Twinrix, and HBVaxPro-40) in healthy nonresponders: a multicentre, open-label, randomised, controlled, superiority trial. Lancet Infect Dis. 2020;20(1):92-101.

23. Cardell K, Akerlind B, Sallberg M, Fryden A. Excellent response rate to a double dose of the combined hepatitis $a$ and $B$ vaccine in previous nonresponders to hepatitis B vaccine. J Infect Dis. 2008;198(3):299-304.

\section{Publisher's Note}

Springer Nature remains neutral with regard to jurisdictional claims in published maps and institutional affiliations.

Ready to submit your research? Choose BMC and benefit from:

- fast, convenient online submission

- thorough peer review by experienced researchers in your field

- rapid publication on acceptance

- support for research data, including large and complex data types

- gold Open Access which fosters wider collaboration and increased citations

- maximum visibility for your research: over $100 \mathrm{M}$ website views per year

At BMC, research is always in progress.

Learn more biomedcentral.com/submissions 